III. Aus der Königlichen Universitätspoliklinik für Halsund Nasenkranke zu Berlin.

\title{
Ein Fall von Struma mit Drehung und Compression der Trachea und Durchbruch in dieselbe. ${ }^{1}$ )
}

Von Dr. Edmund Meyer, Assistenten.

Neubildungen in der Trachea gehören zweifellos zu den seltensten Geschwülsten, die zur Beobachtung kommen. Vielleicht am häufigsten sehen wir die Granulationsgeschwülste der Luftröhre nach der Tracheotomie, und Papillome. In der Litteratur finden sich sodann einige Fälle von Osteomen der Trachea, etwa sieben Fälle von primärem Carcinom derselben und zwei Sarkome. Häufiger finden wir die Trachea secundär von malignen Neubildungen der umgebenden Organe befallen, besonders bei Carcinom des Oesophagus, des Larynx und der Glandula thyreoidea. Ein seltenes Vorkommnis, das von Bruns beschrieben ist, bilden Tumoren der Trachea, die aus abgesprengten Schilddrüsenkeimen besteben. Ein jeder casuistischer Beitrag zur Lehre von den Geschwülsten der Luft. röhre dürfte daher das Interesse ganz besonders in Anspruch nehmen.

Die Krankengeschichte des Patienten, den ich Ihnen vorstellen möchte, ist folgende:

Franz Kage, 52 Jahre alt, Klempner, kam am 30. Mai d. J. wegen Husten, Kratzen im Halse, zeitweise blutigem Auswurf und Athembeschwerden in die Königliche Universitätspoliklinik für Hals- und Nasenkranke. Der Patient giebt an, bereits seit der Jugend einen dicken Hals zu haben. Seit lăngerer Zeit will er bereits ein langsames Wachsen der Geschwulst bemerkt haben, seit einem Jahre etwa soll das Wachsthum rascher fortgeschritten sein. Seit dieser Zeit hat die Athemnoth erheblich zugenommen und in letzter Zeit einen so hohen Grad erreicht, dass Patient gezwungen ist, nachts im Bette zu sitzen.

Patient ist ein ziemlich kräftiger Mann von blasser, leicht eyanotischer Hautfarbe. Puls 76, kräftig, Respiration 24; schon nach geringen Bewegungen ist ein lauter Stridor in- und exspiratorius zu hören. Die rechte Seite des Halses ist von einem Tumor eingenommen, der, aus der Fossa jugularis aufragend, in der Mittellinie nach oben bis zum Bogen des Ringknorpels, nach links bis über die Mittellinie und nach rechts bis beinahe an die Wirbelsäule heranreicht. Der Tumor fühlt sich im allgemeinen hart an, er trägt an seinem vorderen, oberen Rand drei bis wallnussgrosse rundliche Fortsătze, von denen der mittlere deutlich, die beiden seitlichen undeutlicher fluctuiren. Die Haut ist nirgends mit der Geschwulst verwachsen, letztere folgt beim Schlucken den Bewegungen des Kehlkopfes, der nach links herübergedrängt ist. Nase und Rachen des Patienten bieten keine Besonderheiten. Die Schleimhaut des Kehlkopfes ist blass. Die Glottis steht schief.

1) Demonstration in der Berl. laryngol. Gesellschaft am 15. Juli 1892. 
Die Stimmbänder erscheinen leicht geröthet ohne Bewegungsanomalieen. Dicht unterhalb des Larynx sieht man die Trachea nach links gedrängt und um ihre Achse gedreht, ausserdem ist die rechte Trachealwand stark in das Lumen der Trachea hinein vorgewölbt. Der untere Rand der Vorwölbung ist mit dem Auge nicht genau abzugrenzen. Auf der Höhe der Vorwölbung, etwa dem 4.-5. Trachealring entsprechend, sieht man einen mindestens bohnengrossen Tumor, der mit Blut bedeckt ist. Nach Abräuspern desselben präsentirt sich die Oberfläche der Geschwulst glatt, von intensiv rother Farbe. Die Schleimhaut erscheint überall intact, aber leicht blutend. Die Consistenz des Tumors ist ziemlich derb. An der linken Seite des Thorax sieht man eine flache Vorwölbung, welche, etwa in der Mitte des Sternum beginnend, sich nach links bis zur Mitte der Entfernung der Parasternal- und Mammillarlinie erstreckt. Nach unten reicht dieselbe bis zur dritten Rippe. Die Hautvenen der Brustwand sind erweitert und in ihrem Verlaufe geschlängelt. Genau den Grenzen der sichtbaren Vorwölbung entsprechend, ist der Percussionsschall gedämpft, das Athemgeräusch abgeschwächt. Die physikalische Untersuchung ergiebt im übrigen normale Verhältnisse.

Man kann leicht in Versuchung kommen, in unserem Falle in Berücksichtigung des Uebergreifens der Neubildung auf die Luftröhre die Diagnose auf ein Carcinom der Schilddrüse, das sich per continuitatem auf die Trachea fortgesetzt hat, zu stellen. Aber die Entwickelung der Geschwulst spricht nicht für ihre Malignität. Schon in seiner Lehrzeit hat der Patient seine Struma beobachtet und ein allmähliches Wachsthum bis zu ihrer jetzigen Grösse constatirt, allerdings soll die Wachsthumsintensität seit einem Jahre erheblich zugenommen haben. Man könnte nur einwenden, dass es nichts ungewöhnliches ist, dass einfache Strumen in späteren Jahren einen krebsigen Charakter annehmen und von diesem Zeitpunkte an erheblich schneller wachsen. Nun beobachten wir aber den Patienten seit über sechs Wochen, ohne eine Vergrösserung des Tumors am Halse oder in der Trachea feststellen zu können. Auch zum ulcerösen Zerfall besteht entschieden keine Neigung, wie die gänzliche Intactheit des Schleimhautüberzuges zeigt. Endlich mussten wir annehmen, dass ein Carcinom von der Ausdehnung unseres Tumors mit Betheiligung der Trachea nach einem Bestehen von einem Jahre erheblich grössere Kachexie verursacht haben müsste.

Aber was für eine gutartige Geschwulst kann denn überhaupt in unserem Falle vorliegen? Meiner Ansicht nach kann es sich nur um eine Struma handeln, die in die Trachea hineingewuchert ist. Die Fälle gehören allerdings zu den grössten Seltenheiten, es ist bisher nur einer von Paltauf in den Ziegler'schen Beiträgen zur pathologischen Anatomie December 1891 publicirt. Der von Paltauf beschriebene Fall ist in Kürze folgender: ein 29 jähriges Dienstmädchen kommt mit heftigen Athembeschwerden auf die Albert'sche Klinik. Die sofortige Tracheotomie beseitigt die Athemnoth. Patientin wird dann auf die Schrötter'sche Klinik verlegt. Der dort aufgenommene laryngoskopische Befund ist folgender: die Schleimhaut unterhalb des rechten Stimmbandes ist tumorartig vorgewölbt, weich. Die linke Wand erscheint bis zur Höhe der Tracheotomiewunde hereingewölbt, und zwar in nach unten zunehmender Intensität, sodasss die Lichtung bis auf eine kleine Oeffnung an der linken Seite aufgehoben erscheint. Die Dilatation ergab anfangs ein gutes Resultat, bald traten aber Schwellung und Schmerzen auf. Die Incision eines Abscesses brachte keine Erleichterung. Unter Zunahme der Athemnoth trat der Exitus ein. Paltauf machte die Section und unterzog den Tumor einer eingehenden mikroskopischen Untersuchung. Bei derselben stellte es sich heraus, dass der Tumor aus Strumagewebe bestand, das durch die Zwischenmembranen der Knorpelringe hindurchgewachsen war.

Für den Patienten dürfte es im übrigen ziemlich bedeutungslos sein, dass es sich um eine gutartige Neubildung handelt, da an einen operativen Eingriff bei der grossen Ausdehnung der Geschwulst wohl kaum gedacht werden kann. Wir haben uns bisher darauf beschränkt, Jodkali innerlich darzureichen, die Athembeschwerden sollen dabei auch einigermaassen nachgelassen haben. Eine Punction der Cysten wird vielleicht zeitweise noch etwas mehr Erleichterung bringen, trotzdem aber glaube ich, dass die Prognose entschieden ungünstig zu stellen ist.

Nachtrag, den 18. December 1892. Ich habe den Patienten vor zwei Tagen zum letzten male geseheu. Derselbe giebt an, weniger Athembeschwerden zu haben als vor einem halben Jahr zur Zeit, als er unsere Hilfe aufsuchte. Der Tumor aussen am Halse zeigt kein erhebliches Wachsthum, auch die Dämpfungsgrenzen sind nicht verschoben; hingegen ist das Lumen der Trachea noch enger geworden, es erscheint bei der Tracheoskopie auf einen dreieckigen Spalt reducirt. Trotzdem ist der Patient imstande, seiner schwerden Arbeit, er beschäftigt sich hauptsächlich mit Wasserleitungsanlagen, ohne Störung nachzugehen. 